Artículo

\title{
Rentabilidad y rendimiento agronómico de lechuga acuapónica
}

\author{
Mario Francisco García-Zertuche ${ }^{2}$ \\ Alberto Sandoval-Rangel ${ }^{1}$ \\ Valentín Robledo-Torres ${ }^{1}$ \\ Adalberto, Benavides-Mendoza ${ }^{1}$ \\ Armando Robledo-Olivo ${ }^{1}$ \\ Marcelino Cabrera-de la Fuente ${ }^{1 \S}$ \\ ${ }^{1}$ Universidad Autónoma Agraria Antonio Narro. Calzada Antonio Narro núm. 1923, Buenavista, Saltillo, \\ Coahuila, México. CP. 25315. (asandovalr16@gmail.com; robledo3031@gmail.com; \\ abenmen@gmail.com; armando.robledo@outlook.com). ${ }^{2}$ Doctorado en Ciencias en Agricultura Protegida- \\ Universidad Autónoma Agraria Antonio Narro. Calzada Antonio Narro núm. 1923, Buenavista, Saltillo, \\ Coahuila, México. CP. 25315. (mariogarzer@ hotmail.com). \\ ${ }^{\S}$ Autor para correspondencia: cafum@yahoo.com.
}

\section{Resumen}

Con el fin de innovar un sistema de producción acuícola que incremente la productividad del agua, se realizó un experimento en una granja acuícola en Saltillo, Coahuila, donde se estableció el cultivo de lechuga bajo un sistema hidropónico denominado raíz flotante, en condiciones de malla sombra y fertilización foliar, se analizaron las variables agronómicas: longitud de raíz, grosor de tallo, altura, peso fresco, numero de hojas, área foliar y diámetro de corona en cuatro tratamientos distintos y se analizaron distintos indicadores de rentabilidad: valor actual neto, tasa interna de retorno (TIR), relación beneficio costo y retorno sobre la inversión, para determinar la factibilidad económica del sistema, por lo que, el objetivo de esta investigación fue determinar el rendimiento agronómico y la rentabilidad económica del sistema productivo de lechuga raíz flotante en condiciones de malla sombra y fertilización foliar. Los resultados obtenidos en las variables de rendimiento agronómico mostraron incrementos en altura, peso fresco, número de hojas, área foliar y diámetro de corona en comparación con su testigo absoluto de 41.77\%, 113.9\%, 30.43\%, $155.92 \%$ y $22.22 \%$ respectivamente. El análisis de rentabilidad económica muestra resultados favorables para la inversión adicional. Esta investigación demuestra la viabilidad económica y una mejora en la productividad agronómica de la lechuga raíz flotante en condiciones de malla sombra y fertilización foliar.

Palabras clave: acuaponía, malla sombra, producción, raíz flotante, sustentabilidad del agua.

Recibido: marzo de 2021

Aceptado: junio de 2021 


\section{Introducción}

El crecimiento del sector agrícola se basa en la expansión de las áreas de cultivo; sin embargo, hoy en día el potencial para el crecimiento está disminuyendo debido al crecimiento poblacional, disponibilidad de tierras cultivables y la escasez de agua (Wada et al., 2014). Teniendo en cuenta que la agricultura es el principal consumidor de agua a nivel mundial y es la responsable producir el 70\% de los alimentos en el mundo. (Smilovic et al., 2018; Zheng et al., 2018; Kazem, 2020) menciona que existen tres fuentes de pérdida de agua en los riegos agrícolas, la evaporación en la superficie del suelo, por filtración de drenaje (escorrentía y percolación) y las pérdidas por derrame.

Aunado a esto el sector agrícola enfrenta dos grandes desafíos, el estrés hídrico e inseguridad alimentaria y las regiones más afectadas serán las zonas rurales áridas y semiáridas del mundo, donde se practica la agricultura de temporal y el uso del agua para riego se ve limitado por falta de tecnología o recurso económico (Jin et al., 2018; Mandal, 2020). Por lo tanto, se requiere de sistemas de producción alternativos y sustentables que ayuden a optimizar el uso eficiente del agua para la producción de alimentos (Cabrera, 2014; Ríos, 2016; Velazco, 2018).

La integración de sistemas productivos es una alternativa para optimizar el uso de los recursos naturales y generar una producción de alimentos más sustentable (Huong et al., 2018). Los sistemas acuapónicos, por su doble propósito, la producción de peces y hortalizas son actividades sustentables al mejorar la productividad del agua (Mirzoyan et al., 2018; Bosma et al., 2017) mediante el aumento y diversificación de productos. Diversos autores mensionan tener en cuenta que toda actividad de reutilización de agua en cuencas o riegos puede compensar las pérdidas percibidas a escala de campo en términos de cantidad de agua (Gheewala, 2017; Brauman, 2013).

Li et al. (2020) menciona que la productividad del agua se define como la relación entre los beneficios netos de los cultivos y la cantidad de agua utilizada en los diferentes sistemas de producción. Por lo anterior, la acuaponía es considerada como una alternativa para solucionar los problemas de agotamiento de suelos fértiles, disponibilidad de agua, contaminación ambiental y demanda de alimentos (Huong et al., 2018). Las hortalizas de hojas son comunes en estos sistemas, por sus ciclos cortos de producción y bajos requerimientos nutrimentales (Rakocy, 2012).

La lechuga es uno de los vegetales de mayor consumo a nivel mundial (Kim et al., 2016; Yang et al., 2019), se consume en fresco y tiene un gran contenido de nutrientes como vitaminas A, C, E, B1, B2, B3, B9, al igual que fosforo, hierro, calcio, potasio y son ricas en antioxidantes y aminoácidos (Carranza, 2009) en comparación con otros que se cocinan o procesan (Xiao et al., 2012).

Sin embargo, Yagi y Kokubu (2018) mencionan que todo proyecto productivo debe ser respaldado por un análisis de rentabilidad económico financiero, diferentes autores mencionan que por su valoración en unidades monetarias los análisis de rentabilidad económico financiero sirven como fundamento para la toma de decisiones mediante distintos indicadores de factibilidad financiera (Smith et al., 2014; Ofileanu, 2014; Ramli e Iskandar, 2014), siendo el VAN, TIR, RB/C y ROI, los indicadores financieros más comunes para determinar los análisis de rentabilidad económico (Zhao, 2016). Con base a lo anterior y con la finalidad de buscar una solución para mitigar o reducir los problemas que enfrenta en la actualidad el sector agrícola, el objetivo del presente trabajo fue determinar la viabilidad económica y productiva de un sistema acuapónico lechuga raíz flotante en condiciones de malla sombra con fertilización foliar. 


\section{Materiales y métodos}

\section{Localización del experimento}

El experimento fue establecido en la granja de un productor cooperante en el rancho La Joya, ubicado a $25^{\circ} 14^{\prime} 52.1^{\prime \prime}$ latitud norte $101^{\circ} 16^{\prime}$ ' $0.5^{\prime}$ ' longitud oeste, en el ejido de Derramadero, en el municipio de Saltillo, Coahuila, México. La granja cuenta con un sistema de engorda acuícola, constituido por 12 estanques circulares de geomembrana de polietileno de alta densidad (HDPE.75 $\mathrm{Mn}$ ) con esqueleto de soporte metálico, con $10 \mathrm{~m}$ de diámetro y $1.2 \mathrm{~m}$ de altura por estanque para almacenar un volumen de agua de 94248 L en su capacidad máxima por estanque. Dentro de cada estanque se tenían sembrados 2200 alevines de trucha arcoíris (Oncorhynchus mykiss) con la finalidad de producir dos toneladas de trucha por estanque.

\section{Material vegetal}

Se utilizó como material vegetativo lechuga (Lactuca sativa) variedad Climax de la casa comercial Western Seeds. Esta es una lechuga tipo romana que presenta cabezas bien formadas con hojas grandes y envolventes, con un tiempo de siembra a cosecha aproximado de 90 a 95 días.

\section{Desarrollo de plántulas y trasplante}

Las plántulas se establecieron en la Universidad Autónoma Agraria Antonio Narro (UAAAN), ubicada a $25^{\circ} 21^{\prime}$ ' 19" latitud norte, $101^{\circ} 01^{\prime}$ ' 48' longitud oeste, a una altura de $1779 \mathrm{msnm}$ en Buenavista, Saltillo, Coahuila, en el mes de mayo de 2019 en el departamento de Horticultura en un invernadero de mediana tecnología, en charolas de poliestireno de 200 cavidades, utilizando como sustrato peat-moss y perlita en una relación 70/30, una vez brotada la semilla, estas fueron regadas dos veces por día, hasta obtener plántulas con tres hojas verdaderas y una altura de $12 \mathrm{~cm}$ (30 días posteriores a la emergencia), se llevaron a la granja acuícola para su trasplante donde se extrajeron de la charola con todo y cepellón, para posteriormente realizar un lavado de raíces a fin de eliminar el sustrato e incorporarlas al efluente acuícola.

El trasplante fue realizado a los 30 días de haber emergido las plantas, donde se colocaron en placas de unicel de cinco centímetros de espesor y un metro cuadrado, trazando una densidad de población de 20 plantas $\mathrm{m}^{-2}$, en los tanques de geomembrana.

\section{Nutrición vegetal}

La fertilización foliar se realizó tomando como referencia la solución Steiner (1961) rebajada al $75 \%$ y está misma aplicada en diferentes concentraciones en función de la etapa fenológica del cultivo, siguiendo una fertilización foliar al $25 \%$ en su etapa de trasplante, $50 \%$ desarrollo, $75 \%$ en etapa de crecimiento y al $100 \%$ en la etapa final, aplicando cada tercer día después del trasplante.

\section{Tratamientos}

Se analizaron cuatro tratamientos (T) con 10 repeticiones por tratamiento. T1) sin malla sombra (SM) y sin fertilización foliar (SF); T2) sin malla sombra (SM) y con fertilización foliar (CF). T3) con malla sombra $(\mathrm{CM})$ y sin fertilización foliar (SF); T4) con malla sombra (CM) y con fertilización foliar $(\mathrm{CF})$. 


\section{Medición del rendimiento agronómico}

Para la medición de las variables agronómicas, se recolectó todo el material vegetativo del experimento del rancho La Joya, alrededor de las 10:00 am para su medición en campo y posterior mente fue llevado al laboratorio de cultivo de tejidos del departamento de Horticultura en la UAAAN. Las variables evaluadas fueron: longitud de raíz, grosor de tallo, altura de la planta, peso fresco, numero de hojas, área foliar y diámetro de la corona.

Para la medición de longitud de raíz y altura se utilizó una cinta métrica, donde se tomó en cuenta para la medición de la raíz la base de la lechuga hasta la punta de la raíz y para la altura se tomó en cuenta de la base de la lechuga hasta la parte superior de la hoja, el peso fresco se obtuvo de los pesos individuales de las plantas de los distintos tratamientos en la etapa final de cosecha, para lo anterior se utilizó una balanza de la marca And Hr-200 (Max $210 \mathrm{~g} \mathrm{~d}=0.1 \mathrm{mc}$ ), el área foliar se midió al finalizar el estudio con un medidor portátil de área foliar modelo LI-500, el diámetro comercial se consideró la longitud de la circunferencia cultivo en su etapa final al cosechar $(\mathrm{C} / \pi)$ para obtener el área de corona se tomó en cuenta la mitad del diámetro comercial por planta $\left({ }^{*} r^{2}\right)$ y se utilizó un vernier digital de la de marca Steren Her-411 con precisión: $( \pm 0.1 \mathrm{~mm})$ y resolución: $(0.1 \mathrm{~mm})$ Versión 0.0 para medir el grosor de tallo.

\section{Medición de la factibilidad económica}

Se realizó un análisis de factibilidad económica utilizando la metodología de proyecto de inversión y se introdujeron los costos de producción del sistema alternativo malla sombra con fertilización foliar extrapolando la producción a una hectárea, cotizando los productos y equipos utilizados en la zona cercana a la ciudad de Saltillo, Coahuila, mostrado en el Cuadro 1.

\section{Cuadro 1. Costos de producción sistema lechuga acuapónica con malla sombra y fertilización foliar.}

\begin{tabular}{cccc}
\hline Conceptos & Costo unitario & Unidades por hectárea & Costo por hectárea \\
\hline Costo variable & & & \\
Semilla & $\$ 0.0017$ & 200000 & $\$ 1026.00$ \\
Plántulas & $\$ 0.30$ & 199680 & $\$ 179712.00$ \\
Fertilizantes $75 \%$ & $\$ 14.05$ & 40 días d/trasplante & $\$ 1686.00$ \\
Fertilizantes 50\% & $\$ 7.02$ & 70 días d/trasplante & $\$ 1474.20$ \\
Fertilizantes 25\% & $\$ 3.51$ & 90 días d/trasplante & $\$ 947.70$ \\
Aminoácidos & $\$ 800.00$ & $4.25 \mathrm{~L} \mathrm{día}^{-1}(30$ días) & $\$ 306000.00$ \\
Bralic repelente ajo & $\$ 500.00$ & $4.25 \mathrm{~L} \mathrm{día}^{-1}(30$ días) & $\$ 191250.00$ \\
Total costo variable & & & $\$ 682095.90$ \\
Inversión adicional & & & $\$ 1216000.00$ \\
Malla sombra & $\$ 9500.00$ & 128 estructuras & $\$ 59904.00$ \\
Sujetador & $\$ 0.30$ & 199680 & $\$ 648960.00$ \\
Charolas & $\$ 65.00$ & 9984 & $\$ 1924864.00$ \\
Total inv. adicional & & & \\
\hline
\end{tabular}




\begin{tabular}{cccc}
\hline Conceptos & Costo unitario & Unidades por hectárea & Costo por hectárea \\
\hline Costos fijos & & & \\
Gastos administrativos & $\$ 15000.00$ & 12 & $\$ 180000.00$ \\
Gastos de operación & $\$ 31300.00$ & 12 & $\$ 375600.00$ \\
Total costo fijo & & & $\$ 555600.00$
\end{tabular}

Costo total

$\$ 1237695.90$

Nota: los costos de producción del sistema lechuga acuapónica con malla sombra y fertilización foliar se calcularon para tres ciclos productivos en un año, tomando en cuenta los costos de producción como inversión adicional a la granja acuícola de los productores cooperantes se extrapolaron los datos a una hectárea de producción.

\section{Valor actual neto (VAN)}

Para determinar el VAN se utilizó la ecuación: VAN $\left.=-\mathrm{I}_{0}+\sum_{\mathrm{n}=0}^{\mathrm{N}}\left(\frac{\mathrm{Fn}}{(1+\mathrm{r})^{\mathrm{n}}}\right)=-1+\frac{\mathrm{F} 1}{(1+\mathrm{r})}+\frac{\mathrm{F} 2}{(1+\mathrm{r})^{2}}+\frac{\mathrm{Fn}}{(1+\mathrm{r})^{\mathrm{n}}} \quad 1\right)$ propuesta por Stewart et al. (2001). Donde: $-\mathrm{I}_{0}=$ equivale la inversión inicial del proyecto; $\mathrm{Fn}=$ diferencia entre el flujo de efectivo de los ingresos con los gastos en la vida útil del proyecto; $\mathrm{n}=$ vida útil del proyecto; y r= tasa mínima de retorno (TMR) o la tasa de interés que se le atribuye al proyecto.

Para interpretar los resultados de la ecuación del valor actual neto se utiliza la síguete escala: VAN> 0 , el proyecto es aceptado; $\mathrm{VAN}<0$, el proyecto es rechazado; $\mathrm{VAN}=0$, la decisión es indiferente si se acepta o se rechaza.

\section{Tasa interna de retorno (TIR)}

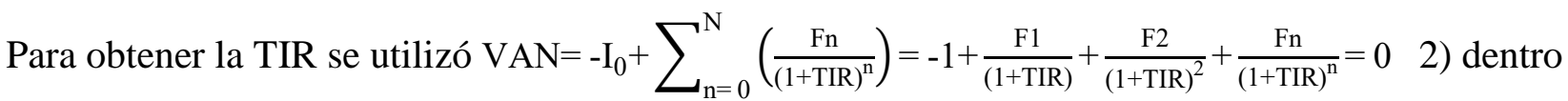
de la ecuación (1) el valor de $r^{*}$ se sustituye por 0 y el valor de la TIR que varía según el porcentaje de utilidad que el inversionista está dispuesto a generar por el uso de su capital en el proyecto (Canales, 2015); para lograr interpretar los resultados de la ecuación de la TIR se utiliza la siguiente escala: TIR $>$ TMR, el proyecto es aceptado; TIR $<$ TMR, el proyecto es rechazado; TIR= TMR, la decisión de aceptar o rechazar el proyecto es indiferente. Mellichamp (2017) menciona que la TIR, una medida de rentabilidad ampliamente utilizada, es la tasa de descuento que arroja valor actual neto $(\mathrm{VAN})=0$ para un flujo de flujos de efectivo positivos y negativos.

\section{Relación beneficio costo $(\mathrm{RB} / \mathrm{C})$}

Para obtener el resultado del indicador relación beneficio costo se dividió el beneficio o utilidad calculada al final del proyecto entre el costo total del proyecto según la ecuación $R_{B / C}=\frac{\sum_{j=0}^{n} \frac{B j}{(1+i)^{n}}}{\sum_{j=0}^{n} \frac{C j}{(1+i)^{j}}}$ 3) utilizada por Daniels et al. (2019). Donde: $\mathrm{Bj}=$ ingresos generados durante la vida útil del proyecto; $\mathrm{Cj}=$ costo generado en el transcurso del proyecto; $\mathrm{j}=$ periodo de tiempo que se está evaluando dentro del proyecto; $\mathrm{i}=$ tasa de interés o la TMR; $\mathrm{y} n=$ tiempo o vida útil del proyecto. 
Para interpretar los resultados del RB/C utilizamos la siguiente escala: $\mathrm{RB} / \mathrm{C}<0$, los costos del proyecto son mayores a los beneficios esperados, se rechaza el proyecto de inversión. RB/C> 0 , los beneficios o utilidades del proyecto son mayores a los costos de inversión, se acepta el proyecto de inversión.

\section{Retorno sobre la inversión (ROI)}

Para obtener el cálculo del indicador retorno sobre la inversión se utilizó la ecuación $\mathrm{ROI}=\frac{\sum_{\mathrm{t}=0}^{\mathrm{T}} \mathrm{Rt} /{ }_{(1+\mathrm{i})^{\mathrm{t}}}^{\mathrm{t}}}{\sum_{\mathrm{t}=0}^{\mathrm{T}} \mathrm{C}_{\mathrm{t}} /(1+\mathrm{i})^{\mathrm{t}}}$ 4). Donde: $\mathrm{Rt}=$ beneficio o utilidad generada a través del tiempo por la inversión realizada; $\mathrm{Ct}=$ costo total de la inversión a través del tiempo; y $\mathrm{i}=$ tasa de interés. Una forma para corroborar el resultado de la ecuación del ROI es la siguiente: donde las utilidades netas son los ingresos menos el costo de producción, con la ecuación $\mathrm{ROI}=(\mathrm{I}-\mathrm{C}) / \mathrm{C} * 100$ 5). Donde: $\mathrm{I}=$ ingreso o utilidad generada; $\mathrm{C}=$ costo de la inversión elaborado. Para interpretar los resultados del ROI utilizamos la siguiente escala: $\mathrm{ROI}<0$, el proyecto de inversión no es rentable. $\mathrm{ROI}>0$, el proyecto de inversión si es rentable. Obteniendo, así como resultado el porcentaje que equivale a la utilidad de la inversión. Kousky (2019) menciona que es una herramienta que se utiliza tradicionalmente en el ámbito privado. Para evaluar y comparar proyectos de inversiones, una manera simple de interpretarlo es las ganancias netas de un proyecto divididas por los costos del proyecto.

\section{Diseño experimental}

El experimento se estableció con un diseño experimental completamente al azar con arreglo factorial $2 \times 2$, donde el primer factor es el uso de malla sombra y el segundo factor es la aplicación de fertilización foliar, sin fertilización (SF) y con fertilización (CF), con un total de cuatro tratamientos $(\mathrm{T})$ y 10 repeticiones por tratamiento.

\section{Análisis estadístico}

Los resultados de cada una de las variables agronómicas evaluadas se analizaron mediante análisis de varianza (Anova), y se aplicó la prueba de diferencia mínima significativa de Fisher para comparar las medias $(p \leq 0.05)$, en el programa estadístico InfoStat/L.

\section{Resultados y discusión}

\section{Análisis de rendimiento agronómico}

\section{Factor malla sombra}

Para la variable de longitud de raíz no hubo diferencias estadísticas entre tratamientos (Cuadro 2), lo que indica en este caso que no se modificó el crecimiento radicular al usar malla sombra; sin embargo, Reyes (2016) menciona que depende mucho del cultivo acuapónico que se esté desarrollando al igual que el sistema acuapónico con el que se esté trabajando. 
Cuadro 2. Interacción entre ambientes de producción para variables agronómicas de lechuga cultivadas en un sistema acuapónico con malla sombra y fertilización foliar.

\begin{tabular}{|c|c|c|c|c|c|c|c|c|}
\hline \multicolumn{2}{|c|}{ Factor } & $\begin{array}{l}\text { Longitud de } \\
\text { raíz }\end{array}$ & $\begin{array}{l}\text { Grosor } \\
\text { de tallo }\end{array}$ & Altura & $\begin{array}{l}\text { Peso } \\
\text { fresco }\end{array}$ & $\begin{array}{l}\text { Núm. } \\
\text { hojas }\end{array}$ & $\begin{array}{l}\text { Área } \\
\text { foliar }\end{array}$ & $\begin{array}{l}\text { Diámetro } \\
\text { de corona }\end{array}$ \\
\hline \multicolumn{2}{|c|}{ Sin malla } & $22.38 \mathrm{a}$ & $1.76 \mathrm{a}$ & $6.44 \mathrm{~b}$ & $37.62 \mathrm{~b}$ & $11.5 \mathrm{~b}$ & $39.16 b$ & $9 \mathrm{~b}$ \\
\hline \multicolumn{2}{|c|}{ Con malla } & $21.06 \mathrm{a}$ & $1.59 \mathrm{~b}$ & $9.13 \mathrm{a}$ & $80.47 \mathrm{a}$ & $15 \mathrm{a}$ & $100.22 \mathrm{a}$ & $11 \mathrm{a}$ \\
\hline \multicolumn{2}{|c|}{ Sin fertilización } & $21.56 \mathrm{a}$ & $1.73 \mathrm{a}$ & $7.19 \mathrm{~b}$ & $54.74 \mathrm{a}$ & $13 \mathrm{a}$ & $65.63 \mathrm{a}$ & $9.88 \mathrm{a}$ \\
\hline \multicolumn{2}{|c|}{ Con fertilización } & $21.88 \mathrm{a}$ & $1.63 \mathrm{a}$ & $8.38 \mathrm{a}$ & $63.34 \mathrm{a}$ & $13.5 \mathrm{a}$ & $73.75 \mathrm{a}$ & $10.13 \mathrm{a}$ \\
\hline SM & $\mathrm{SF}$ & $21.63 \mathrm{a}$ & $1.83 \mathrm{a}$ & $5.63 \mathrm{c}$ & $34.84 \mathrm{~b}$ & $11.5 \mathrm{~b}$ & $36.13 b$ & $8.88 \mathrm{~b}$ \\
\hline SM & $\mathrm{CF}$ & $23.13 \mathrm{a}$ & $1.7 \mathrm{a}$ & $7.25 \mathrm{~b}$ & $40.4 \mathrm{~b}$ & $11.5 \mathrm{~b}$ & $42.19 \mathrm{~b}$ & $9.13 \mathrm{ab}$ \\
\hline $\mathrm{CM}$ & $\mathrm{SF}$ & $21.5 \mathrm{a}$ & $1.63 \mathrm{~b}$ & $8.75 \mathrm{a}$ & $74.64 \mathrm{a}$ & $14.5 \mathrm{a}$ & $95.13 \mathrm{a}$ & $10.88 \mathrm{ab}$ \\
\hline $\mathrm{CM}$ & $\mathrm{CF}$ & $20.63 \mathrm{a}$ & $1.55 \mathrm{~b}$ & $9.5 \mathrm{a}$ & $86.29 \mathrm{a}$ & $15.5 \mathrm{a}$ & $105.31 \mathrm{a}$ & $11.13 \mathrm{a}$ \\
\hline
\end{tabular}

$\mathrm{SM}=$ sin malla sombra; $\mathrm{CM}=$ con malla sombra; $\mathrm{SF}=$ sin fertilización foliar; $\mathrm{CF}=$ con fertilización foliar, los valores con diferentes literales son significativamente diferentes (Fisher LSD $\leq 0.05$ ).

Los resultados del análisis estadístico muestran para la variable grosor de tallo que el testigo absoluto (T1) tiene 10.69\% más que el T4 el cual se encontraba en malla sombra y con fertilización foliar. Choosakul (2017) obtuvo resultados similares donde el grosor del tallo de sus lechugas también es mayor en la lechuga cultivada sin malla sombra.

Para las variables altura, peso fresco, numero de hojas, área foliar y diámetro de corona el T4 muestra diferencia estadística en comparación con el testigo absoluto, siendo 41.77\%, 113.9\%, $30.43 \%, 155.92 \%$ y $22.22 \%$ respectivamente mayor que el T1, diferentes autores como, Espinoza et al. (2016); Ayala et al. (2011) reportaron efectos positivos en altura con el uso de malla sombras en chile manzano (Capsicum pubescebc) y tomate (Solanum lycopersicum) respectivamente, Gaurav et al. (2016) menciona que las plantas cultivadas bajo malla sombra producen mayor biomasa y follaje que las cultivadas en campo abierto.

Choosakul (2017) en su investigación menciona que el área foliar de las hojas de lechuga que se encontraban en malla sombras de diferentes colores se registró más grandes que las que estaban en condiciones de campo abierto. Fu et al. (2012), señala que la alta intensidad de la luz promueve el crecimiento de la lechuga; sin embargo, este efecto promotor del crecimiento se satura a un cierto nivel de intensidad de luz y los rangos recomendados para la producción de lechuga son de 400 a $600 \mu \mathrm{mol} \mathrm{m} \mathrm{m}^{-2}$.

\section{Factor de fertilización foliar}

Para el factor de fertilización foliar no se presentaron diferencias significativas (Cuadro 2), en las variables agronómicas evaluadas de longitud de raíz, grosor de tallo, peso fresco, numero de hojas, área foliar, diámetro de corona, Nozzi et al. (2018) obtuvo resultados similares donde al desarrollar un estudio con diferentes aplicaciones de nutrición foliar a un sistema de lechuga raíz flotante no obtuvieron diferencias significativas entre tratamientos, al igual que Pickens (2015) reportó que no hubo diferencias significativas entre tratamientos al evaluar tomates cherry (Solanum lycopersicum var. cerasiforme) regados con efluente acuícola y fertilización foliar. 
Sin embargo, para la variable altura el T4 mostró una diferencia de $16.55 \%$ en com paración con el testigo absoluto, lo que coincide con Roosta and Hamidpour (2011, 2012), donde mencionan que el suplemento de nutrientes de forma foliar en sistemas acuapónicos mejora la productividad de las plantas.

\section{Factor malla y fertilización}

En la interacción de factores (Cuadro 2), para la variable de longitud de raíz no se observan diferencias estadísticas, sin embargo para las variables grosor de tallo, altura, peso fresco, numero de hojas, área foliar y diámetro de corona se obtuvieron diferencias estadísticas entre tratamientos que se debieron a que la malla sombra que favoreció el crecimiento de los cultivos, a lo que Mudau et al. (2017) menciona en su trabajo realizado con espinacas (Spinacia oleracea L.) que el crecimiento vegetativo de los cultivos se ve más pronunciado en plantas cultivadas bajo malla sombras en comparación con plantas cultivadas en campo abierto, también Choosakul (2017) menciona que el uso de malla sombra de cualquier tipo de color logra aumentar el crecimiento de los cultivos.

Por otra parte, el uso de malla sombra con aplicaciones foliares incremento el diámetro de corona, lo que Yep et al. (2019) menciona que el suplemento de nutrientes de forma foliar si mejora la productividad de las plantas en sistemas acuapónicos, debido a que se suministra de forma adicional los elementos faltantes en los efluentes acuícolas.

\section{Análisis económico}

El análisis económico fue realizado en un escenario con bajo precio de venta para tener panorama pesimista y poder ofrecer una mejor alternativa en la toma de decisiones, para el cálculo de vida útil del proyecto se estableció un panorama a seis años descrito en el (Cuadro 3), siendo este factible con los resultados de los diferentes indicadores positivos.

Cuadro 3. Análisis de rentabilidad económico del sistema productivo.

\begin{tabular}{|c|c|c|c|c|c|c|c|}
\hline Seis años & 0 & 1 & 2 & 3 & 4 & 5 & 6 \\
\hline Plantas & & 199680 & 199680 & 199680 & 199680 & 199680 & 199680 \\
\hline $\begin{array}{l}\text { Precio de } \\
\text { venta }\end{array}$ & & $\$ 9.50$ & $\$ 9.50$ & $\$ 9.50$ & $\$ 9.50$ & $\$ 9.50$ & $\$ 9.50$ \\
\hline Ingresos & & $\$ 1896960.00$ & $\$ 1896960.00$ & $\$ 1896960.00$ & $\$ 1896960.00$ & $\$ 1896960.00$ & $\$ 1896960.00$ \\
\hline $\begin{array}{l}\text { Costo } \\
\text { fijo }\end{array}$ & & $\$ 555600.00$ & $\$ 583380.00$ & $\$ 612549.00$ & $\$ 643176.45$ & $\$ 675335.27$ & $\$ 709102.04$ \\
\hline $\begin{array}{l}\text { Costo } \\
\text { variable }\end{array}$ & & $\$ 682095.90$ & $\$ 716200.70$ & $\$ 752010.73$ & $\$ 789611.27$ & $\$ 829091.83$ & $\$ 870546.42$ \\
\hline $\begin{array}{l}\text { Costo } \\
\text { total }\end{array}$ & & $\$ 1237695.90$ & $\$ 1299580.70$ & $\$ 1364559.73$ & $\$ 1432787.72$ & $\$ 1504427.10$ & $\$ 1579648.46$ \\
\hline Saldo & & $\$ 659264.10$ & $\$ 597379.31$ & $\$ 532400.27$ & $\$ 464172.28$ & $\$ 392532.90$ & $\$ 317311.54$ \\
\hline $\begin{array}{l}\text { Flujo } \\
\text { efectivo }\end{array}$ & $-\$ 1924864.00$ & $\$ 659264.10$ & $\$ 597379.31$ & $\$ 532400.27$ & $\$ 464172.28$ & $\$ 392532.90$ & $\$ 317311.54$ \\
\hline VAN & $\$ 47908.30$ & & & & & & \\
\hline
\end{tabular}




\begin{tabular}{|c|c|c|c|c|c|c|c|}
\hline Seis años & 0 & 1 & 2 & 3 & 4 & 5 & 6 \\
\hline \multirow[t]{4}{*}{ TIR } & $16 \%$ & VPN & $\$ 0.00$ & $\begin{array}{c}\Sigma \text { Ingreso } \\
\text { neto }\end{array}$ & $\$ 1972772.30$ & TI & $15 \%$ \\
\hline & & & & $\begin{array}{c}\Sigma \text { Ingreso } \\
\text { bruto }\end{array}$ & $\$ 7179012.29$ & & \\
\hline & & $\mathrm{R} \mathrm{B/C}$ & 1.379 & $\Sigma$ Costos & $\$ 5206239.99$ & ROI & $\$ 1.02$ \\
\hline & & & & $\begin{array}{c}\Sigma \mathrm{C}+ \\
\text { Inversión }\end{array}$ & $\$ 7131103.99$ & & \\
\hline
\end{tabular}

Nota: el precio de venta fue el peor de los escenarios posibles para la venta de lechuga en el periodo.

La TIR solo es $1 \%$ mayor al TMR o tasa de interés y la $\mathrm{RB} / \mathrm{C}$ de 1.38 quiere decir que por cada peso invertido en el proyecto se generan 0.38 centavos, al igual que el ROI por cada peso invertido el retorno de la inversión es de 0.02 centavos, siendo estos resultados bajos significando que el riesgo es mayor al ser un resultado que está en los límites aceptables, esto se debe al panorama pesimista en el cual desarrollamos en análisis económico y la vida útil del proyecto.

\section{Conclusiones}

El cultivo de lechuga acuaponica en condiciones de raíz flotante y malla sombra, mostró ser más eficiente en cuanto al desarrollo del cultivo presentando incrementos en el rendimiento agronómico.

El sistema lechuga raíz flotante en condiciones de malla sombra y fertilización foliar, mostró ser rentable a través de los diferentes indicadores económicos.

No se observó influencia de la interacción fertilización foliar y la malla sombra en las diferentes variables de rendimiento evaluadas, por lo anterior se puede lograr ser más rentable al bajar los costos variables de producción omitiendo el gasto por fertilización foliar, lo que muestra una eficiencia productiva en el uso del efluente acuícola como nutrición para el cultivo de lechuga, es importante resaltar, que el sistema lechuga raíz flotante en condiciones de malla sombra presenta una factibilidad económica positiva en una vida útil de seis años, además la lechuga que proviene de sistemas en condiciones de agricultura protegida se caracteriza por tener un sobreprecio y es de mayor aceptación en el mercado.

\section{Literatura citada}

Ayala-Tafoya, F.; Zatarain-López, D.; Valenzuela-López, M.; Partida-Ruvalcaba, L.; VelázquezAlcaraz, T.; Díaz-Valdés, T. y Osuna-Sánchez, J. A. 2011. Crecimiento y rendimiento de tomate (Solanum lycopersicum) en respuesta a radiación solar transmitida por mallas sombra. Terra Latinoam. 29(4):403-410.

Bosma, R. H.; Lacambra, L.; Landstra, Y.; Perini, C.; Poulie, J.; Schwaner, M. J. and Yin, Y. 2017. The financial feasibility of producing fish and vegetables through aquaponics. Aquacultural Engineering. 78(part B):146-154.

Brauman, A; Siebert, S. and Foley, A. 2013. Improvements in crop water productivity increase water sustainability and food security a global analysis. Environmental Research Letters, $8(2): 24-30$. 
Cabrera, R. 2014. Alternative water sources for urban landscape irrigation in arid regions. J. Arid Land Studies. 24(1):89-92.

Canales, S. y Ricardo, J. 2015. Criterios para la toma de decisión de inversiones. Departamento de Economía UNAM-Managua. Revista Electrónica de Investigación en Ciencias Económicas. 3(5):101-117

Carranza, C.; Lanchero, O.; Miranda, D. y Chaves, B. 2009. Análisis del crecimiento de lechuga (Lactuca sativa L.) "Batavia" cultivada en un suelo salino de la Sabana de Bogotá. Agronomía Colombiana. 27(1):41-48.

Choosakul, N. and Pagamas, P. 2017. The influence of color shading net on the growing of lettuce. Appl. Mechanics and Materials. 866:33-36.

Daniels, S.; Martensen, H.; Schoeters, A.; Van-Berghe, W.; Papadimitriou, E.; Ziakopoulos, A. and Perez, O. M. 2019. A systematic cost-benefit analysis of 29 road safety measures. Accident Analysis \& Prevention. 133:105-292.

Espinoza-Torres, L. y Ramírez-Abarca, O. 2016. Rentabilidad de chile manzano (Capsicum pubescebc R Y P) producido en invernadero en Texcoco, Estado de México. Rev. Mex. Cienc. Agríc. 7(2):325-335.

Fu, W.; Li, Y. and Wu. 2012. Effects of different light intensities on chlorophyll fluorescence characteristics and yield in lettuce. Sci. Hortic. 135:45-51.

Gaurav, A.; Raju, D.; Janakiram, T.; Singh, B.; Jain, R. and Krishnan, S. 2016. Effect of different coloured shade nets on production and quality of cordyline. Indian J. Agric. Sci. 86(7):865-869.

Gheewala, S.; Silalertruksa, T.; Nilsalab, P.; Lecksiwilai, N.; Sawaengsak, W.; Mungkung, R. and Ganasut J. (2017). Water stress index and its implication for agricultural land-use policy in Thailand. Inter. J. Environ. Sci. Technol. 15(4):833-846.

Huong, N.; Huu, C. T.; Thi, N. T. T. and Lebailly, P. 2018. efficiency of different integrated agriculture aquaculture systems in the red River Delta of Vietnam. Sustainability. 10(2):1-13.

Jin, N.; Ren, W.; Tao, B.; He, L.; Ren, Q.; Li, S. and Yu, Q. 2018. Effects of water stress on water use efficiency of irrigated and rainfed wheat in the Loess Plateau, China. Sci. Total. Environ. 642:1-11.

Kazem-Attar, H.; Noory, H.; Ebrahimian, H. and Liaghat, M. 2020. Efficiency and productivity of irrigation water based on water balance considering quality of return flows. Agric. Water Manag. 231(106025):1-10.

Kim, M.; Moon, Y.; Tou, J.; Mou, B. and Waterland, N. 2016. Nutritional value, bioactive compounds and health benefits of lettuce (Lactuca sativa L.). J. Food Composition and Analysis. 49:19-34.

Kousky, C.; Ritchie, L.; Tierney, K. and Lingle, B. 2019. Return on investment analysis and its applicability to community disaster preparedness activities. Calculating costs and returns. Inter. J. Disaster Risk Reduction. 41(101296):1-9.

Li, M.; Xu, Y.; Fu, Q.; Singh, V. P.; Liu, D. and Li, T. 2020. Efficient irrigation water allocation and its impact on agricultural sustainability and water scarcity under uncertainty. J. Hydrology. 586(124888):1-12.

Mandal, S.; Vema, K.; Kurian, C. and Sudheer, P. 2020. Improving the crop productivity in rainfed areas with water harvesting structures and deficit irrigation strategies. J. Hydrology. 586(124818):1-51.

Mellichamp, D. 2017. Internal rate of return: good and bad features, and a new way of interpreting the historic measure. Computers \& Chemical Engineering. 106(17):396-406. 
Mirzoyan, N.; Avetisyan, N.; Mnatsakanyan, H. and Tadevosyan, L. 2018. Groundwater use and efficiency in small and medium-sized aquaculture farms in Ararat Valley, Armenia. Groundwater for Sustainable Development. 6:1-5.

Mudau, A.; Soundy, P. and Mudau, F. 2017. Response of baby spinach (Spinacia oleracea L.) to photoselective nettings on growth and postharvest quality. HortScience. 52(5):719-724.

Nozzi, V.; Graber, A.; Schmautz, Z.; Mathis, A. and Junge, R. 2018. Nutrient management in aquaponics: comparison of three approaches for cultivating lettuce, mint and mushroom Herb. Agronomy. 8(27):1-15.

Ofileanu, D. and Bumbescu, S. 2014. The analysis of the factors which influence the change of the breakeven point. Procedia Economics and Finance. 16:356-367.

Pickens, J. M. 2015. Integrating effluent from recirculating aquaculture systems with greenhouse cucumber and tomato production. Doctoral dissertation, Auburn University. http://hdl.handle.net/10415/4782.

Rakocy, J.; Masser, M. and Losordo, T. 2012. Recirculating aquaculture tank production systems: aquaponics integrating fish and plant culture. southern regional aquaculture center. USDA 454:1-16.

Rakocy, J. E. 2012. Aquaponics-integrating fish and plant culture. aquaculture production systems. 344-386 pp.

Ramli, I. and Iskandar, D. 2014. Control authority, business strategy, and the characteristics of management accounting information systems. Procedia Social and Behavioral Sciences. 164:384-390.

Reyes-Flores, M.; Sandoval-Villa, M.; Rodríguez-Mendoza. N.; Trejo-Téllez, L.; SánchezEscudero, J. y Reta-Mendiola, J. 2016. Concentración de nutrientes en efluente acuapónico para producción de Solanum lycopersicum L. Rev. Mex. Cien. Agríc. 16(Esp.):3529-3542.

Ríos-Flores, J.; Torres-Moreno, M.; Ruiz-Torres, J. y Torres-Moreno, M. 2016. Eficiencia y productividad del agua de riego en trigo (Triticum vulgare) de Ensenada y Valle de Mexicali, Baja California, México. Acta Universitaria. 26(1):20-29

Roosta, H. R. and Hamidpour, M. 2011. Effects of foliar application of some macro- and micronutrients on tomato plants in aquaponic and hydroponic systems. Sci. Hortic. 122(3):396-402.

Roosta, H. R. and Mohsenian, Y. 2012. Effects of foliar spray of different Fe sources on pepper (Capsicum annum L.) plants in aquaponic system. Sci. Hortic. 146:182-191.

Smilovic, M.; Gleeson, T.; Adamowski, J. and Langhorn, C. 2018. More food with less waterOptimizing agricultural water use. Adv. Water Res. 123:256-26.

Smith, M. T.; Schroenn, G. J. and Blignaut, J. N. 2014. The financial and economic feasibility of rural household biodigesters for poor communities in South Africa. Waste Management. 34:352-362.

Steiner, A. 1961. A universal method for preparing nutrient solutions of a certain desired composition. Plant Soil. 15:134-154.

Stewart, J.; Allison, N. and Johnson, S. 2001. Putting a price on biotechnology. Nature Biotechnol. 19(9):813-817

Velasco-Muñoz, J.; Aznar-Sánchez, J.; Belmonte-Ureña, L. and Román-Sánchez, I. 2018. Sustainable water use in agriculture: a review of worldwide research. Sustainability. 10(1084):1-18.

Wada, Y.; Wisser, D. and Bierkens, M. 2014. Global modeling of withdrawal, allocation and consumptive use of surface water and groundwater resources. Earth System Dynamics. $5: 15-40$. 
Xiao, Z.; Lester, G.; Luo, Y. and Wang, Q. 2012. Assessment of vitamin and carotenoid concentrations of emerging food products: edible microgreens. J. Agric. Food Chem. 60:7644-7651.

Yagi, M. and Kokubu, K. 2018. Corporate material flow management in Thailand: the way to material flow cost accounting. J. Cleaner Production. 198:763-775.

Yang, T. and Kim, H. J. 2019. Nutrient management regime affects water quality, crop growth, and nitrogen use efficiency of aquaponic systems. Sci. Hortic. 256:108619.

Yep, B. and Zheng, Y. 2019. Aquaponic trends and challenges A review. J. Cleaner Production. 228:1586-1599.

Zhao, X.; Yao, G. and Tyner, W. E. 2016. Quantifying breakeven price distributions in stochastic techno-economic analysis. Applied Energy. 183:318-326.

Zheng, H.; Bian, Q.; Yin, Y.; Ying, H.; Yang, Q. and Cu, Z. 2018. Closing water productivity gaps to achieve food and water security for a global maize supply. Scientific Reports. 8(14762):14762. 